\title{
Severe vitamin D deficiency is not related to SARS-CoV-2 infection but may increase mortality risk in hospitalized adults: a retrospective case-control study in an Arab Gulf country
}

\author{
Abdullah M. Alguwaihes ${ }^{1}$. Shaun Sabico ${ }^{2} \cdot$ Rana Hasanato $^{3} \cdot$ Mohammed E. Al-Sofiani $^{1,4,5} \cdot$ Maram Megdad $^{6}$. \\ Sakhar S. Albader ${ }^{7}$ - Mohammad H. Alsari ${ }^{7}$. Ali Alelayan ${ }^{7}$. Ebtihal Y. Alyusuf ${ }^{1}$. Saad H. Alzahrani ${ }^{8}$. \\ Nasser M. Al-Daghri ${ }^{2}$ Anwar A. Jammah ${ }^{1}$
}

Received: 12 February 2021 / Accepted: 5 March 2021 / Published online: 31 March 2021

(c) The Author(s), under exclusive licence to Springer Nature Switzerland AG 2021

\begin{abstract}
Purpose As the world continues to cautiously navigate its way through the coronavirus disease 2019 (COVID-19) pandemic, several breakthroughs in therapies and vaccines are currently being developed and scrutinized. Consequently, alternative therapies for severe acute respiratory coronavirus 2 (SARS-CoV-2) prevention, such as vitamin D supplementation, while hypothetically promising, require substantial evidence from countries affected by COVID-19. The present retrospective casecontrol study aims to identify differences in vitamin D status and clinical characteristics of hospitalized patients screened for SARS-CoV-2, and determine associations of vitamin D levels with increased COVID-19 risk and mortality.

Methods A total of 222 [SARS-CoV-2 (+) N= 150 (97 males; 53 females); SARS-CoV-2 (-) N=72 (38 males, 34 females)] out of 550 hospitalized adult patients screened for SARS-CoV-2 and admitted at King Saud University Medical City-King Khalid University Hospital (KSUMC-KKUH) in Riyadh, Saudi Arabia from May-July 2020 were included. Clinical, radiologic and serologic data, including 25(OH)D levels were analyzed.

Results Vitamin D deficiency $(25(\mathrm{OH}) \mathrm{D}<50 \mathrm{nmol} / \mathrm{l})$ was present in $75 \%$ of all patients. Serum $25(\mathrm{OH}) \mathrm{D}$ levels were significantly lower among SARS-CoV-2 (+) than SARS-CoV-2 (-) patients after adjusting for age, sex and body mass index (BMI) $(35.8 \pm 1.5 \mathrm{nmol} / \mathrm{l}$ vs. $42.5 \pm 3.0 \mathrm{nmol} / 1 ; p=0.037)$. Multivariate regression analysis revealed that significant predictors for SARS-CoV-2 include age $>60$ years and pre-existing conditions $(p<0.05)$. Statistically significant predictors for mortality adjusted for covariates include male sex [Odds ratio, OR 3.3 (95\% confidence interval, CI 1.2-9.2); $p=0.02$ ], chronic kidney disease [OR 3.5 (95\% CI 1.4-8.7); $p=0.008$ ] and severe 25(OH)D deficiency $(<12.5 \mathrm{nmol} / \mathrm{l})$, but at borderline significance [OR 4.9 (95\% CI (0.9-25.8); $p=0.06]$.

Conclusion In hospital settings, 25(OH)D deficiency is not associated with SARS-CoV-2 infection, but may increase risk for mortality in severely deficient cases. Clinical trials are warranted to determine whether vitamin $\mathrm{D}$ status correction provides protective effects against worse COVID-19 outcomes.
\end{abstract}

Keywords Vitamin D · COVID-19 · Case-control · Saudi Arabia · Severe vitamin D deficiency

AbdullahM. Alguwaihes and Shaun Sabico contributed equally.

Abdullah M. Alguwaihes

aalguwaihes@ksu.edu.sa

1 Division of Endocrinology, Department of Internal Medicine, College of Medicine, King Saud University, Riyadh, Saudi Arabia

2 Biochemistry Department, College of Science, King Saud University, Riyadh, Saudi Arabia

3 Pathology Department, College of Medicine, King Saud University, Riyadh, Saudi Arabia
4 Division of Endocrinology, Diabetes and Metabolism, The John Hopkins University, Baltimore, MD, USA

5 Strategic Center for Diabetes Research, College of Medicine, King Saud University, Riyadh, Saudi Arabia

6 General Directorate of Health Affairs in Eastern Province, Dammam, Saudi Arabia

7 King Saud University Medical City, Riyadh, Saudi Arabia

8 Obesity, Endocrine and Metabolism Center, King Fahad Medical City, Riyadh, Saudi Arabia 


\section{Background}

There is universal acceptance that the exponential spread of severe acute respiratory syndrome coronavirus 2 (SARSCoV-2), the novel pathogen responsible for coronavirus disease 2019 (COVID-19), had instantaneously and irreversibly disrupted normalcy both at an individual level and most, if not all of the global affairs for the year 2020. At present, majority of the people with COVID-19 eventually recover, given its mild to moderate course. Interestingly, approximately $45 \%$ of infected individuals are considered 'asymptomatic' and have been deemed 'silent carriers' of the pandemic [1]. Compared to its predecessors, SARS$\mathrm{CoV}-2$ is much more contagious, but with a lower death rate (2.3\%) than SARS-CoV (9.5\%) and even lower than the Middle Eastern Respiratory Syndrome (MERS-CoV) coronavirus (34.4\%) [2]. In all coronavirus epidemics, management has been largely supportive. Nevertheless, and in the case of COVID-19, potential therapeutics for those with severe symptoms, such as convalescent plasma [3] and remdesivir [4], have been explored with promising results. In parallel, the race to provide effective vaccines are also being expeditiously developed for COVID-19 prevention [5], with the one from Pfizer ${ }^{\circledR}$ currently gaining attraction for mass production after a series of successful clinical trials [6]. Among the roster of potential therapies, one alternative treatment that has been widely accepted and in fact, included in the rapid COVID-19 management is the use of vitamin $\mathrm{D}$, largely driven by empirical evidence on the association of vitamin $\mathrm{D}$ to viral infections [7, 8].

Vitamin D is a secosteroid pro-hormone with a wellestablished role in maintaining calcium homeostasis. More recently, vitamin $\mathrm{D}$ has gained prominence for its pleiotropic and extra-skeletal effects [9]. It is estimated that globally, more than a billion people have vitamin D deficiency, with the highest prevalence mostly coming from countries in the Middle East, including Saudi Arabia [10, 11]. Currently, vitamin $\mathrm{D}$ has established potency against several viral respiratory infections and has consistently been observed to accelerate healing of injured tissues, primarily the lungs [12], considered compelling reasons why vitamin D merits further exploration in its preventive role in COVID-19. Furthermore, accumulating evidence from retrospective studies done mostly in Europe [13, 14], USA [15] and China [16] indicate that lower vitamin D levels were commonly observed among hospitalized patients with confirmed COVID-19, and may have some role in determining the severity and outcome of COVID-19. To date, only two studies have been conducted in the Middle East to examine the association between vitamin D levels and risk of acquiring, and dying from, COVID-19. One large-scale study in Israel has reported vitamin D deficiency as an independent risk factor of COVID-19 infection in the general population [17]; and the retrospective study in Iran which reported that low vitamin $\mathrm{D}$ levels are associated with severe lung damage and higher mortality among hospitalized COVID-19 patients [18]. So far, no published studies have examined the link between vitamin D deficiency and risk of COVID-19 infection or mortality in Saudi Arabia or any Arabian Gulf countries. Data from this part of the world, which is notoriously underrepresented in medical literature, are of clinical value, given its geographic and ethnic differences as compared to other regions. In addition, the high prevalence of vitamin $\mathrm{D}$ deficiency in this homogenous population may offer a unique insight into the link between vitamin D levels and COVID-19 that is unique from that reported in other parts of the world.

To fill this gap, the present retrospective, case-control study aims to examine the association between vitamin D levels and clinical profiles of hospitalized individuals screened for SARS-CoV-2, and whether low vitamin D levels are associated with increased risk of COVID-19 infection and mortality in this subpopulation.

\section{Methods}

\section{Study design and participants}

In this single-center retrospective study conducted from May to July, 2020, medical records of 550 adult residents of Riyadh, KSA, who were swabbed for SARS-CoV-2 [N=439 SARS-CoV-2 (+) and $N=111$ SARS-CoV-2 (-)] and consequently admitted at KSUMC-KKUH from May-July 2020, were included [19]. Children and pregnant women were excluded.

\section{SARS-CoV-2 screening}

Diagnosis of SARS-CoV-2 infection was based on national guidelines [20]. Swab samples were obtained from the patient's nasopharyngeal and oropharyngeal orifice/s and sent to the Biosafety Level 2-facility (BSL-2) with Biological Safety Cabinet Class II (BSC-II) in KSUMC, Riyadh, KSA, following universal precautions, for reverse transcription polymerase chain reaction (RT-PCR) analysis. RNA amplification was performed using ViiA7 RT-PCR (Thermo Fisher Scientific, Waltham, MA, USA) and Altona reagents (Altona Diagnostics, Hamburg, Germany). COVID19 Test validation was done through Chemagic ${ }^{\text {TM }}$ 360-D (PerkinElmer, MA, USA). Final interpretation was based on cycle threshold (CT) values obtained from specimens using ViiA7 software. Assay specificity and sensitivity obtained were $100 \%$ based on 23 specimens. Sample investigations 
were performed by certified laboratory personnel following manufacturer's recommendations for the defined cut-off cycle threshold (CT) value for each target gene.

\section{Data collection}

Clinical information included demographics (age and sex), medical history (known cases of diabetes mellitus (DM), hypertension (HPN), cardiovascular disease (CVD), congestive heart failure (CHF), chronic kidney disease (CKD), stroke as well as smoking history) and list of medications $[\beta$-blockers, angiotensin converting enzyme (ACE) inhibitors, angiotensin receptor blockers (ARB), calcium channel blockers (CCB), statins, insulin, oral hypoglycemic, anticoagulants and anti-platelets] were noted on admission. Height and weight were recorded and body mass index $\left(\mathrm{kg} / \mathrm{m}^{2}\right)$ was calculated. Obesity was defined as having a $\mathrm{BMI} \geq 30 \mathrm{~kg} / \mathrm{m}^{2}$. Radiological findings, if available, were recorded.

\section{Serological tests}

Non-fasting and fasting blood samples were obtained in all admitted patients. All samples were analyzed in the KKUHKSUMC central laboratory, a participating and accredited member of the College of American Pathologists (CAP) Laboratory Accreditation Program. Laboratory investigations included glycemic [glycated hemoglobin (HbA1c), random and fasting blood glucose (RBG and FBG, respectively)], lipids [triglycerides, low-density lipoprotein (LDL-) and high-density lipoprotein (HDL)-cholesterol)] profiles of liver [alanine transferase (ALT), aspartate aminotransferase (AST) and lactate dehydrogenase (LDH)], renal [blood urea nitrogen (BUN) and creatinine] and inflammatory markers [ferritin, erythrocyte sedimentation rate (ESR) and C-reactive protein (CRP)] if available, were obtained. Serum 25(OH)D was assessed using CDC-approved vitamin D total assay kits (ADVIA Centaur ${ }^{\circledR}$, Siemens Healthcare GmbH, Erlangen, Germany) as certified by the Vitamin D Standardization-Certification Program (VDSCP) [21], with an assay range of 9.3 to $375 \mathrm{nmol} / \mathrm{l}$. For the purpose of this study, patients whose $25(\mathrm{OH}) \mathrm{D}$ levels were not assessed $(N=328)$, were excluded. The remaining 222 patients $(N=135$ males and $N=87$ females) were included in the data analysis. A flowchart of participants is provided in Fig. 1. Vitamin D deficiency $[25(\mathrm{OH}) \mathrm{D}<50 \mathrm{nmol} / \mathrm{l}]$ and severe vitamin $\mathrm{D}$ deficiency $[25(\mathrm{OH}) \mathrm{D}<12.5 \mathrm{nmol} / \mathrm{l}]$ were defined based on national and regional recommendations [22, 23].

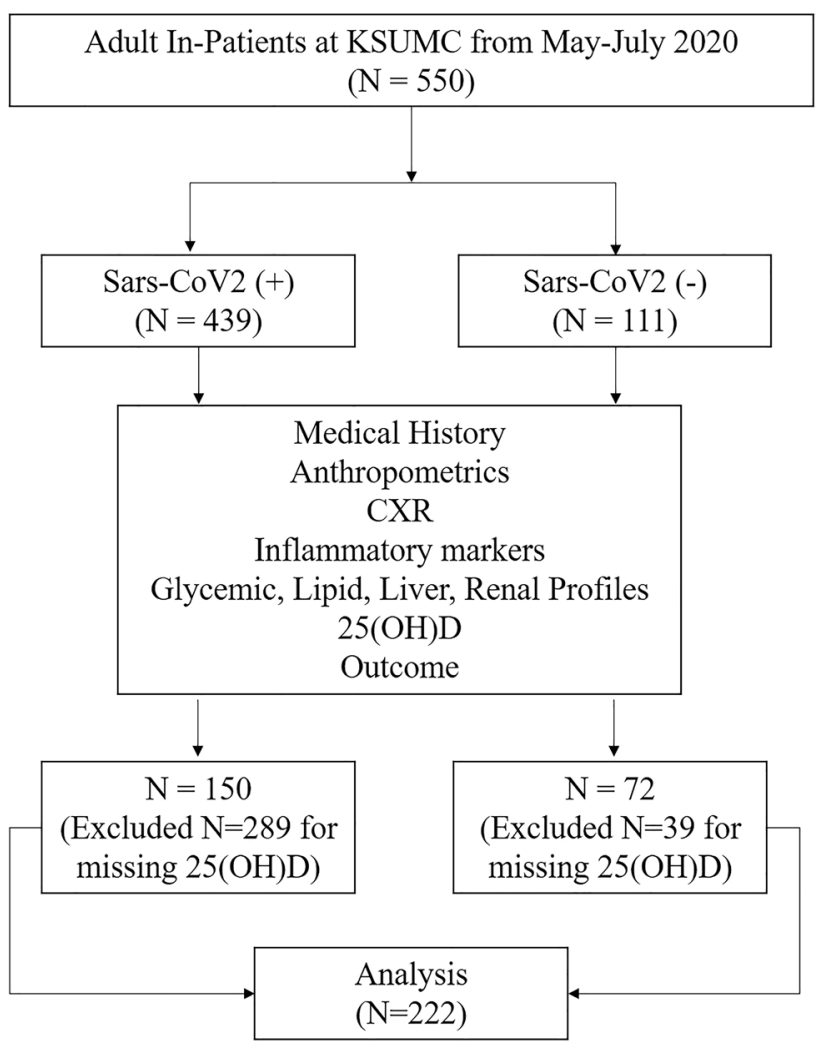

Fig. 1 Flowchart of participants

\section{Data analysis}

Data were analyzed using SPSS version 21.0 (IBM, Chicago, IL, USA). Categorical variables were presented as frequencies $(N)$ and percentages (\%). Continuous variables were presented as mean \pm standard error (SE). Differences in categorical variables according to SARS-CoV-2 status were compared using Chi-Square test. Comparisons between continuous variables according to SARS-CoV-2 status were done using independent Student $T$ test for normally distributed data and Mann-Whitney $U$ test for non-normally distributed data. Analysis of covariance (ANCOVA) was used to obtain $p$ values after adjusting with age, sex and BMI. Multivariate logistic regression analysis was done to determine independent predictors for outcomes of interest [death and SARS-CoV-2(+)] using age, sex and BMI as covariates. Significance was set at $p<0.05$.

Post hoc power analysis was done using the $G^{*}$ Power software [24]. While the given the sample size and obtained $25(\mathrm{OH}) \mathrm{D}$ levels between groups yield $100 \%$ power to detect significant difference, the study was underpowered to detect an association between severe $25(\mathrm{OH}) \mathrm{D}$ deficiency and COVID-19 mortality, having only $21 \%$ power (odds ratio of 1.45 ), given $\alpha=0.05$. 
Table 1 General characteristics of patients

\begin{tabular}{|c|c|c|c|c|}
\hline \multirow[t]{2}{*}{ Parameters } & \multirow[t]{2}{*}{ All } & \multicolumn{2}{|c|}{ SARS-CoV-2 Status } & \multirow[t]{2}{*}{$p$ value } \\
\hline & & Positive & Negative & \\
\hline$N(\%)$ & 222 & 150 & 72 & \\
\hline $\operatorname{Sex}(M / F)$ & $135 / 87$ & $97 / 53$ & $38 / 34$ & \\
\hline Age (years) & $56.6 \pm 16.2$ & $55.5 \pm 15.8$ & $59.1 \pm 16.8$ & 0.12 \\
\hline BMI $\left(\mathrm{kg} / \mathrm{m}^{2}\right)$ & $29.6 \pm 7.7$ & $29.6 \pm 6.7$ & $29.7 \pm 9.4$ & 0.93 \\
\hline \multicolumn{5}{|l|}{ Nationality (\%) } \\
\hline Saudi & $134(60.4)$ & $76(50.7)$ & $58(80.6)$ & \multirow[t]{2}{*}{0.004} \\
\hline Non-Saudi & $88(39.6)$ & $74(49.3)$ & $14(19.4)$ & \\
\hline \multicolumn{5}{|c|}{ Pre-existing conditions (\%) } \\
\hline Obesity & $84(37.8)$ & $57(38)$ & $27(37.5)$ & 0.99 \\
\hline Hypertension & $121(54.5)$ & $68(45.3)$ & $53(73.6)$ & $<0.001$ \\
\hline Diabetes mellitus & $114(47.3)$ & $68(45.4)$ & $46(63.8)$ & 0.02 \\
\hline $\begin{array}{l}\text { Cardiovascular } \\
\text { disease }\end{array}$ & $34(15.3)$ & $15(10)$ & $19(26.4)$ & 0.003 \\
\hline $\begin{array}{l}\text { Congestive heart } \\
\text { failure }\end{array}$ & $27(12.2)$ & $5(3.3)$ & $22(30.6)$ & $<0.001$ \\
\hline $\begin{array}{l}\text { Chronic kidney } \\
\text { disease }\end{array}$ & 39 (17.6) & $15(10)$ & $24(33.3)$ & $<0.001$ \\
\hline Stroke & $19(8.6)$ & $10(6.7)$ & $9(12.5)$ & 0.20 \\
\hline Smoking & $6(2.7)$ & $1(0.9)$ & $5(6.9)$ & $<0.001$ \\
\hline $\begin{array}{l}25(\mathrm{OH}) \\
\mathrm{D}<50 \mathrm{nmol} / \mathrm{l}\end{array}$ & $166(75.1)$ & $117(78)$ & $49(69)$ & 0.18 \\
\hline \multicolumn{5}{|l|}{ Medications (\%) } \\
\hline$\beta$-Blockers & $54(24.3)$ & $24(16.0)$ & $30(41.7)$ & $<0.001$ \\
\hline ACE inhibitors & $33(14.9)$ & $17(11.3)$ & $16(22.2)$ & 0.04 \\
\hline $\mathrm{ARB}$ & $29(13.1)$ & $22(14.7)$ & $7(9.7)$ & 0.40 \\
\hline CCB & $69(31.1)$ & $40(26.7)$ & $29(40.3)$ & 0.04 \\
\hline Statins & $77(34.7)$ & $45(30)$ & $32(44.4)$ & 0.03 \\
\hline Insulin & $46(20.7)$ & $22(14.7)$ & $24(33.3)$ & 0.002 \\
\hline Oral hypoglycemic & $78(35.1)$ & $55(36.7)$ & $23(31.9)$ & 0.55 \\
\hline Anti-coagulants & $11(5.0)$ & $6(4.0)$ & $5(7.0)$ & 0.50 \\
\hline Anti-platelets & $63(28.4)$ & $38(25.3)$ & $25(34.7)$ & 0.16 \\
\hline
\end{tabular}

Significant at $p<0.05$

\section{Results}

Table 1 shows the differences in the characteristics of hospitalized patients according to SARS-CoV-2 status. Overall, more than $60 \%$ of the patients were males. Males were also more common among those who tested positive (65\%) and slightly higher in the negative group (52.8\%) (not shown in table). No significant differences were observed in age and BMI. A significantly higher prevalence of Saudis tested negative as opposed to other nationalities $(p=0.004)$. In terms of pre-existing conditions, the negative group had a significantly higher prevalence of hypertension $(p<0.001)$, $\mathrm{DM}(p=0.02), \mathrm{CVD}(p=0.003), \mathrm{CHF}(p<0.001), \mathrm{CKD}$ $(p<0.001)$ and smoking $(p<0.001)$ than the positive group. In terms of medications, the negative group was also more likely to use $\beta$-blocker $(p<0.001)$, ACE inhibitors $(p=0.04), \mathrm{CCB}, p=0.04)$, statins $(p=0.03)$ and insulin $(p=0.002)$. While vitamin $\mathrm{D}$ deficiency was highly prevalent in all patients (75.1\%), no significant differences were seen between groups. The rest of the comparisons were not significant (Table 1).

Table 2 shows the unadjusted and adjusted differences in radiologic and laboratory investigations of admitted patients based on SARS-CoV-2 status. Majority of those who tested positive presented with bilateral lung infiltrates (69.3\%) which was significantly higher than those who tested negative $(38.5 \%)(p<0.001)$. The positive group also had significantly worse liver and lipid profiles than the negative group in terms of higher AST, LDH and triglycerides (adjusted $p$ values $0.01,<0.001$ and 0.007 , respectively). On the other hand, the negative group had significantly higher levels of BUN and creatinine than the positive group (adjusted $p$ values 0.001 and $<0.001$, respectively). With regard to inflammatory profile, only CRP levels were significantly higher in the positive than the negative group (adjusted $p=0.03$ ). The positive group also had significantly higher $\mathrm{HbAlc}$ and FBG than the negative group (adjusted $p$ values both at 0.02 ). (Table 2). Lastly, the positive group had significantly lower circulating $25(\mathrm{OH}) \mathrm{D}$ levels compared to the negative group, both in unadjusted $(p=0.025)$ and adjusted $(p=0.037) p$ values. In terms of outcome, however, no significant differences were seen in the vitamin D status of those discharged versus those who died (Fig. 2).

In terms of clinical outcomes, no significant differences in groups were observed, with the exception of discharged patients, being significantly higher in the positive than the negative group ( 81.4 versus $65.3 \%$; $p=0.01$ ) (Table 3 ).

In the last Table 4, predictors for SARS-CoV-2 (+) and death were determined using multivariate logistic regression analysis. Age above 60 years, presence of bilateral lung infiltrates and most pre-existing conditions (DM, HPN, CVD, $\mathrm{CHF}$ and CKD) were significantly associated with higher risk for SARS-CoV-2 even after adjusting for covariates ( $p$ values $<0.05$ ). Both vitamin D deficiency at the mild [Odds ratio, OR 1.6 (95\% confidence interval, CI 0.8-3.0)] and severe levels [OR 5.3 (95\% CI 0.6-45.7)] also showed increased risk but were not statistically significant in both unadjusted $(p=0.10)$ and adjusted $(p=0.18)$ models. For death, significant predictors included male sex [OR 1.4 (0.63-3.1); $p=0.02$ ], CVD [OR $2.5(1.0-6.3) ; \mathrm{p}=0.048$ ], CKD [OR 3.2 (1.3-7.6); $p=0.009$ ] and severe vitamin D deficiency [OR $4.7(1.0-21.3) ; \mathrm{p}=0.04]$. CKD was the only significant predictor for death independent of age, sex and BMI [OR 3.5 (1.4-8.7); adjusted $p=0.008$ ]. Worthy to note is that severe vitamin $\mathrm{D}$ deficiency was also associated with mortality but at borderline significance [OR 4.9 $(0.9-25.8) ; p=0.06]$. The rest of the odds ratios and are shown in Table 4. 
Table 2 Clinical characteristics of Covid-19 patients on admission

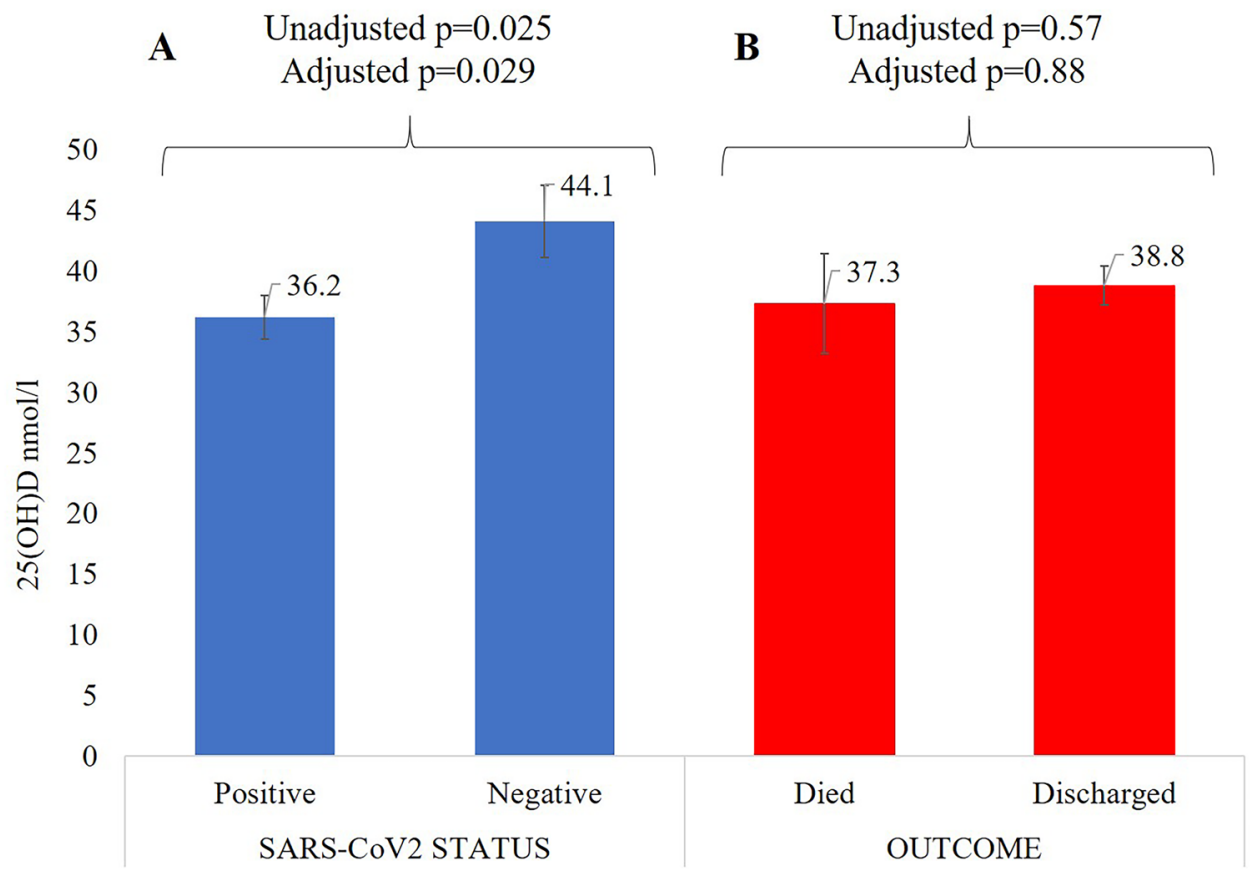

\begin{tabular}{|c|c|c|c|c|}
\hline \multirow[t]{2}{*}{ Parameters } & \multicolumn{2}{|c|}{ SARS-CoV-2 status } & \multicolumn{2}{|l|}{$p$ value } \\
\hline & Positive & Negative & Unadjusted & Adjusted \\
\hline$N$ & 150 & 72 & & \\
\hline \multicolumn{5}{|l|}{ Chest X-ray } \\
\hline $\begin{array}{l}\text { No infiltrates } \\
\text { Unilateral infiltrates } \\
\text { Bilateral infiltrates }\end{array}$ & $\begin{array}{l}29(19.3) \\
17(11.3) \\
104(69.3)\end{array}$ & $\begin{array}{l}22(42.3) \\
10(19.2) \\
20(38.5)\end{array}$ & $<0.001$ & $<0.001$ \\
\hline \multicolumn{5}{|l|}{ Liver profile } \\
\hline $\operatorname{ALT}(\mathrm{U} / \mathrm{l})(20-65)^{\#}$ & $52.9 \pm 3.9$ & $43.4 \pm 7.7$ & $<0.001$ & 0.63 \\
\hline $\operatorname{AST}(\mathrm{U} / \mathrm{l})(15-37)^{\#}$ & $52.3 \pm 3.2$ & $33.2 \pm 3.9$ & $<0.001$ & 0.01 \\
\hline $\mathrm{LDH}(\mathrm{U} / \mathrm{l})(84-246)^{\#}$ & $452.1 \pm 15.0$ & $300.5 \pm 18.4$ & $<0.001$ & $<0.001$ \\
\hline \multicolumn{5}{|l|}{ Renal profile } \\
\hline $\mathrm{BUN}(\mathrm{mmol} / \mathrm{l})(2.5-6.4)^{\#}$ & $8.9 \pm 0.7$ & $13.9 \pm 1.3$ & $<0.001$ & 0.001 \\
\hline Creatinine $(\mu \mathrm{mol} / \mathrm{l})(49-90)^{\#}$ & $160.1 \pm 17.8$ & $331.1 \pm 50.3$ & 0.001 & $<0.001$ \\
\hline \multicolumn{5}{|l|}{ Lipid profile } \\
\hline Triglycerides (mmol/l) & $2.0 \pm 0.1$ & $1.4 \pm 0.1$ & 0.004 & 0.007 \\
\hline HDL-cholesterol (mmol/l) & $0.9 \pm 0.08$ & $1.1 \pm 0.08$ & 0.16 & 0.18 \\
\hline LDL-cholesterol (mmol/l) & $2.0 \pm 0.1$ & $1.9 \pm 0.2$ & 0.55 & 0.80 \\
\hline \multicolumn{5}{|l|}{ Inflammatory profile } \\
\hline Ferritin $(\mu \mathrm{g} / \mathrm{ml})(13-150)^{\#}$ & $1000.9 \pm 106.9$ & $601.9 \pm 149.4$ & $<0.001$ & 0.11 \\
\hline $\operatorname{ESR}(\mathrm{mm} / \mathrm{h})(0-24)^{\#}$ & $76.8 \pm 2.7$ & $73.4 \pm 4.8$ & 0.64 & 0.23 \\
\hline $\mathrm{CRP}(\mathrm{mg} / \mathrm{l})(<10.0)^{\#}$ & $110.4 \pm 6.3$ & $79.2 \pm 11.9$ & $<0.001$ & 0.03 \\
\hline \multicolumn{5}{|l|}{ Glycemic profile } \\
\hline $\mathrm{HbA1c}(\%)$ & $7.8 \pm 0.2$ & $7.1 \pm 0.2$ & 0.01 & 0.02 \\
\hline FBG (mmol/l) & $9.7 \pm 0.4$ & $7.8 \pm 0.5$ & 0.004 & 0.02 \\
\hline $\mathrm{RBG}(\mathrm{mmol} / \mathrm{l})$ & $9.9 \pm 0.4$ & $11.3 \pm 0.9$ & 0.18 & 0.17 \\
\hline \multicolumn{5}{|l|}{$25(\mathrm{OH}) \mathrm{D}$} \\
\hline 25(OH)D (nmol/l) (75-250) & $35.8 \pm 1.5$ & $42.5 \pm 3.0$ & 0.025 & 0.037 \\
\hline
\end{tabular}

\#Non-normal variables; covariates include age, sex and BMI; significant at $p<0.05$
Fig. 2 Differences in 25(OH)D levels according to a SARSCoV-2 Status and $\mathbf{b}$ Outcome of hospitalized patients. Covariates for adjusted $p$ value include age, sex and BMI 
Table 3 Differences in outcomes

\begin{tabular}{lllll}
\hline Outcome (\%) & All & \multicolumn{2}{l}{ SARS-CoV-2 status } & $p$ value \\
\cline { 3 - 4 } & & Positive & Negative & \\
\hline$N$ & 222 & 150 & 72 & \\
Intubated & $30(13.6)$ & $24(16.0)$ & $6(8.3)$ & 0.14 \\
Admitted in ICU & $60(27.3)$ & $46(30.7)$ & $14(19.4)$ & 0.08 \\
Discharged & $165(76.0)$ & $118(81.4)$ & $47(65.3)$ & 0.01 \\
Death & $28(12.8)$ & $21(14.0)$ & $7(9.7)$ & 0.39 \\
\hline
\end{tabular}

Data presented as $N(\%)$; significant at $p<0.05$

\section{Discussion}

While the quest for effective therapies and vaccines against COVID-19 is still on-going with modest success, remedies aimed at SARS-CoV-2 prevention remain particularly imperative as the world learns to coexist with the pandemic. In the present retrospective, case-control study, results from the clinical data of hospitalized patients in Riyadh, Saudi Arabia indicate a very high prevalence of vitamin D deficiency over-all, and significantly lower 25(OH)D levels among SARS-CoV-2-positive as compared to SARS-CoV-2-negative patients. This statistical significance persisted even after adjusting for age, sex and BMI. Furthermore, severe vitamin $\mathrm{D}$ deficiency appears to be an independent predictor for COVID-19 mortality by as much as 5 times compared to those whose $25(\mathrm{OH}) \mathrm{D}$ levels were higher. The present study is one of the few to use confirmed SARS-CoV-2-negative patients as controls, and the first in the Arabian Gulf region.
The significantly lower 25(OH)D levels among hospitalized COVID-19 patients are in harmony with other case-control findings obtained in Spain [13] and China [16], although the controls used in the mentioned studies were apparently healthy individuals from population-based cohorts that were matched for age and sex. It also supports the growing evidence linking vitamin $\mathrm{D}$ deficiency with severity of COVID-19 outcomes [25-27]. A big difference in the present study as compared to other case-control investigations is that all patients were not only confirmed hospital admissions, but all have been screened for SARS-CoV-2 before stratification to positive and negative groups in the same time frame, potentially narrowing selection bias which is a limitation of previous studies.

In the present study, all the pre-existing conditions where vitamin $\mathrm{D}$ deficiency is known to be prevalent (DM, HPN, CVD, CHF, CKD and stroke) were significant predictors for COVID-19 infection independent of age, sex and BMI. Furthermore, CKD was an important predictor of COVID-19 mortality in the present cohort even after adjusting for established covariates. While severe vitamin D deficiency was a significant predictor of death only in the unadjusted model, the risk is nevertheless elevated even in the adjusted models, albeit at borderline significance. CKD, and kidney diseases in general, impairs vitamin D catabolism [28]. Levels of circulating 25(OH)D were observed to substantially fall parallel to decreasing glomerular filtration rate (GFR) [29]. Severe vitamin D deficiency can therefore be linked to COVID-19 mortality indirectly, depending on the extent of renal damage and existing renal disorders. The absence of significant risk in

Table 4 Significant predictors of SARS-CoV-2 (+) and death using multivariate logistic regression analysis

\begin{tabular}{|c|c|c|c|c|c|c|c|c|}
\hline \multirow[t]{3}{*}{ Predictor } & \multicolumn{4}{|c|}{ SARS-CoV-2(+) } & \multicolumn{4}{|l|}{ Death } \\
\hline & \multicolumn{2}{|l|}{ Unadjusted } & \multicolumn{2}{|l|}{ Adjusted } & \multicolumn{2}{|l|}{ Unadjusted } & \multicolumn{2}{|l|}{ Adjusted } \\
\hline & OR $(95 \% \mathrm{CI})$ & $p$ value & OR $(95 \% \mathrm{CI})$ & $p$ value & OR $(95 \% \mathrm{CI})$ & $p$ value & OR $(95 \% \mathrm{CI})$ & $p$ value \\
\hline Age $>60$ years & $1.9(1.1)$ & 0.03 & & & $1.4(0.63-3.1)$ & 0.41 & & \\
\hline Male & $1.6(0.9-2.9)$ & 0.09 & & & $3.3(1.2-9.2)$ & 0.02 & & \\
\hline $\mathrm{BMI}>30 \mathrm{~kg} / \mathrm{m}^{2}$ & $1.0(0.6-1.8)$ & 0.99 & & & $0.7(0.3-1.6)$ & 0.39 & & \\
\hline Smoking & $12.5(0.8-186)$ & 0.07 & $12.8(0.8-210)$ & 0.08 & $1.2(0.1-13.2)$ & 0.85 & $1.7(0.2-20.0)$ & 0.66 \\
\hline Bilateral infiltrates & $3.9(1.9-8.2)$ & $<0.001$ & $3.6(1.7-7.1)$ & 0.001 & $3.2(0.9-11.2)$ & 0.07 & $2.9(0.8-10.9)$ & 0.11 \\
\hline $\mathrm{DM}$ & $2.1(1.2-3.8)$ & 0.01 & $2.1(1.1-4.0)$ & 0.02 & $1.6(0.7-3.6)$ & 0.25 & $1.7(0.7-4.0)$ & 0.26 \\
\hline HPN & $3.4(1.8-6.2)$ & $<0.001$ & $3.2(1.6-6.4)$ & 0.001 & $1.2(0.5-2.6)$ & 0.69 & $1.0(0.4-2.6)$ & 0.99 \\
\hline CVD & $3.2(1.5-6.8)$ & 0.002 & $3.2(1.4-7.2)$ & 0.004 & $2.5(1.0-6.3)$ & 0.048 & $2.3(0.8-6.1)$ & 0.10 \\
\hline $\mathrm{CHF}$ & $12.8(4.6-36)$ & $<0.001$ & $13.8(4.7-39.9)$ & $<0.001$ & $1.7(0.6-4.8)$ & 0.35 & $1.4(0.4-4.3)$ & 0.58 \\
\hline CKD & $4.5(2.2-9.2)$ & $<0.001$ & $3.8(1.8-8.2)$ & 0.001 & $3.2(1.3-7.6)$ & 0.009 & $3.5(1.4-8.7)$ & 0.008 \\
\hline Stroke & $2.0(0.8-5.2)$ & 0.15 & $1.7(0.6-4.9)$ & 0.29 & $2.3(0.7-7.5)$ & 0.18 & $2.0(0.5-7.1)$ & 0.35 \\
\hline $25(\mathrm{OH}) \mathrm{D}<50 \mathrm{nmol} / \mathrm{l}$ & $1.6(0.8-3.0)$ & 0.15 & $1.4(0.7-2.8)$ & 0.30 & $1.2(0.5-2.9)$ & 0.67 & $1.3(0.5-3.2)$ & 0.61 \\
\hline $25(\mathrm{OH}) \mathrm{D}<12.5 \mathrm{nmol} / \mathrm{l}$ & $5.3(0.6-45.7)$ & 0.10 & $4.5(0.5-39.4)$ & 0.18 & $4.7(1.0-21.3)$ & 0.04 & $4.9(0.9-25.8)$ & 0.06 \\
\hline
\end{tabular}

Covariates entered in the model: sex, age and BMI; significant at $p<0.05$ 
mortality observed in SARS-CoV-2-positive patients with $\mathrm{DM}$ alone and/or obesity alone has been documented in similar retrospective studies done within the Arab Gulf region [19, 30], and suggests that the increased risk for COVID-19 death is multifactorial and is probably associated more on the cumulative effects of DM and/or obesity clustering with other chronic diseases, or cardiometabolic multi-morbidity, which are exacerbated in acute complications [31].

Finally, it is worthy to note that more men had COVID19 than women in the present study, confirming other retrospective studies done in Saudi Arabia where males also had worse outcomes than their female counterparts [19, 30]. The male sex being an independent risk factor for SARS-CoV-2 can be attributed to many factors, but mostly for the lack of immune-boosting estrogen. 17 $\beta$-estradiol, in particular, a female hormone in which biosynthesis is regulated by vitamin D via calcium homeostasis [32], has many physiologic actions in terms of the RAS system activation, anti-inflammation, anti-viral protection and endolysosomal degradation [33].

The authors acknowledge some limitations. The lack of statistical significance in eliciting an association between severe vitamin D deficiency and mortality was clearly due to low sample size. Furthermore, the findings only apply to hospitalized patients with pre-existing conditions and not the general community to eliminate selection bias (Berkson's). This is especially true in the present study, given the nature of $25(\mathrm{OH}) \mathrm{D}$ as a negative acute-phase reactant for both acute and chronic inflammatory-state diseases $[34,35]$. Having mentioned such, it is nevertheless safe to assume that the majority of the participants already had vitamin D deficiency, given their age and pre-existing conditions, all of which are associated with chronic, lowgrade systematic inflammation [36]. Lastly, the nature of the retrospective design and the small number of admitted patients swabbed made it difficult to properly match the positive and negative cases, hence the discrepancy in the presence of pre-existing conditions. Nevertheless, the study is the first of its kind to compare the clinical characteristics of hospitalized patients screened for SARSCOV2 in the Gulf region, and to demonstrate differences and associations of vitamin D levels to COVID-19 and mortality among hospitalized patients.

In summary, the prevalence of vitamin $\mathrm{D}$ deficiency among hospitalized patients screened for SARS-CoV-2 in Saudi Arabia is remarkably high and is significantly lower in SARS-CoV-2-positive patients, even after adjusting for covariates known to influence vitamin D. Severe vitamin D deficiency, however, is not associated with SARS-CoV-2 infection and is more related to increased mortality risk. The present findings support large-scale clinical trials to confirm whether manipulating vitamin D status through supplementation offers beneficial effects in COVID-19 prevention and treatment, particularly among high-risk individuals living in regions where both vitamin D deficiency and SARS-CoV-2 infection are highly prevalent.

Acknowledgements The authors thank all the residents and nurses of KSUMC-KKUH for support.

Author contributions Conceptualization, AMA and SS; Methodology, SS, MEA, and AAJ; Investigation and data curation, MEA, AAJ, EYA, MM, SSA, MHA, AA, and SHA; writing —original draft preparation, SS; formal analysis, SS; supervision, AMA, NMA and AAJ; funding acquisition, NMA. All authors have read and agreed to the published version of the manuscript.

\section{Declarations}

Conflict of interest The authors declare no conflict of interest.

Statement of human and animal rights All procedures reported comply with the ethical standards of the relevant national and institutional committees on human experimentation and with the Helsinki Declaration of 1975 , as revised in 2008 .

Ethical approval Ethical approval and waiver of informed consent were obtained from the Institutional Review Board (IRB) of the College of Medicine in King Saud University (KSU) in Riyadh, Saudi Arabia (E-20-5090/July 5, 2020).

Informed consent Not applicable.

\section{References}

1. Oran DP, Topol EJ (2020) Prevalence of asymptomatic SARSCoV-2 infection: a narrative review. Ann Intern Med 173:362-367

2. Petrosillo N, Viceconte G, Ergonul O et al (2020) COVID-19, SARS and MERS: are they closely related? Clin Microbiol Infect 26:729-734

3. Thijssen M, Devos T, Ejtahed HS et al (2020) Convalescent plasma against COVID-19: a broad-spectrum therapeutic approach for emerging infectious diseases. Microorganisms 8:E1733

4. Zhao M, Zhang J, Li H et al (2020) Recent progress of antiviral therapy for coronavirus disease 2019. Eur J Pharmacol 173646

5. Graham BS (2020) Rapid COVID-19 vaccine development. Science 368:945-946

6. Sahin U, Muik A, Derhovanessian E et al (2020) COVID-19 vaccine BNT162b1 elicits human antibody and $\mathrm{T}_{\mathrm{H}} 1 \mathrm{~T}$ cell responses. Nature 586:594-599

7. Beard JA, Bearden A, Striker R (2011) Vitamin D and the antiviral state. J Clin Virol 50:194-200

8. Teymoori-Rad M, Shokri F, Salimi V et al (2019) The interplay between vitamin D and viral infections. Rev Med Virol 29:e2032

9. Bouillon R, Marcocci C, Carmeliet G et al (2019) Skeletal and extraskeletal actions of vitamin D: current evidence and outstanding questions. Endocr Rev 40:1109-1151

10. Palacios C, Gonzalez L (2014) Is vitamin D deficiency a major global public health problem? J Steroid Biochem Mol Biol 144:138-145 
11. Al-Daghri NM (2018) Vitamin D in Saudi Arabia: prevalence, distribution and disease associations. J Steroid Biochem Mol Biol 175:102-107

12. Mohan M, Cherian JJ, Sharma A (2020) Exploring links between vitamin D deficiency and COVID-19. PLoS Pathog 16:e1008874

13. Hernández JL, Nan D, Fernandez-Ayala M et al (2020) Vitamin D status in hospitalized patients with SARS-CoV-2 infection. J Clin Endocrinol Metab. https://doi.org/10.1210/clinem/dgaa733

14. Panagiotou G, Tee SA, Ihsan Y et al (2020) Low serum 25-hydroxyvitamin D (25[OH]D) levels in patients hospitalized with COVID-19 are associated with greater disease severity. Clin Endocrinol (Oxf). https://doi.org/10.1111/cen.14276

15. Meltzer DO, Best TJ, Zhang H et al (2020) Association of Vitamin D status and other clinical characteristics with COVID-19 test results. JAMA Netw Open 3:e2019722

16. Ye K, Tang F, Liao X et al (2020) Does serum vitamin D level affect COVID-19 infection and its severity?-a case-control study. J Am Coll Nutr. https://doi.org/10.1080/07315724.2020. 1826005

17. Merzon E, Tworowski D, Gorohovski A et al (2020) Low plasma $25(\mathrm{OH})$ vitamin $\mathrm{D}$ level is associated with increased risk of COVID-19 infection: an Israeli population-based study. FEBS J. https://doi.org/10.1111/febs.15495

18. Abrishami A, Dalili N, Mohammadi Torbati P et al (2020) Possible association of vitamin D status with lung involvement and outcome in patients with COVID-19: a retrospective study. Eur J Nutr. https://doi.org/10.1007/s00394-020-02411-0

19. Alguwaihes AM, Al-Sofiani ME, Megdad M et al (2020) Diabetes and Covid-19 among hospitalized patients in Saudi Arabia: a single-centre retrospective study. Cardiovasc Diabetol 19:205

20. Saudi Center for Disease Prevention and Control (Weqaya). Laboratory diagnosis of COVID-19 in suspected cases. https://covid 19.cdc.gov.sa/professionals-health-workers/laboratory-diagnosis/. Accessed 16 Nov 2020

21. VDSCP Vitamin D Certified Assays. Certifications from 2020 (updated September 2020). https://www.cdc.gov/labstandards/pdf/ hs/CDC_Certified_Vitamin_D_Procedures-508.pdf. Accessed 10 Dec 2020

22. Al-Daghri NM, Al-Saleh Y, Aljohani N et al (2017) Vitamin D status correction in Saudi Arabia: an experts' consensus under the auspices of the European Society for Clinical and Economic Aspects of Osteoporosis, Osteoarthritis, and Musculoskeletal Diseases (ESCEO). Arch Osteoporos 12:1. https://doi.org/10.1007/ s11657-016-0295-y

23. Al Saleh Y, Beshyah SA, Hussein W et al (2020) Diagnosis and management of vitamin D deficiency in the Gulf Cooperative Council (GCC) countries: an expert consensus summary statement from the GCC vitamin D advisory board. Arch Osteoporos $15: 35$
24. Faul F, Erdfelder E, Lang AG et al (2007) G*Power 3: a flexible statistical power analysis program for the social, behavioral, and biomedical sciences. Behav Res Methods 39:175-191

25. Luo X, Liao Q, Shen Y et al (2020) Vitamin D deficiency is inversely associated with COVID-19 incidence and disease severity in Chinese People. J Nutr. https://doi.org/10.1093/jn/nxaa332

26. Baktash V, Hosack T, Patel N et al (2020) Vitamin D status and outcomes for hospitalised older patients with COVID-19. Postgrad Med J. https://doi.org/10.1136/postgradmedj-2020-138712

27. Daneshkhah A, Agrawal V, Eshein A et al (2020) Evidence for possible association of vitamin D status with cytokine storm and unregulated inflammation in COVID-19 patients. Aging Clin Exp Res 32:2141-2158

28. Bosworth C, de Boer IH (2013) Impaired vitamin D metabolism in CKD. Semin Nephrol 33:158-168

29. Nigwekar SU, Tamez H, Thadhani RI (2014) Vitamin D and chronic kidney disease-mineral bone disease (CKD-MBD). Bonekey Rep 3:498

30. Sheshah E, Sabico S, Albakr RM et al (2020) Prevalence of diabetes, management and outcomes among Covid-19 adult patients admitted in a specialized tertiary hospital in Riyadh, Saudi Arabia. Diabetes Res Clin Pract. https://doi.org/10.1016/j.diabres.2020. 108538

31. Apicella M, Campopiano MC, Mantuano M et al (2020) COVID19 in people with diabetes: understanding the reasons for worse outcomes. Lancet Diabetes Endocrinol 8:782-792

32. Kinuta K, Tanaka H, Moriwake T et al (2000) Vitamin D is an important factor in estrogen biosynthesis of both female and male gonads. Endocrinology 141:1317-1324

33. Khan N (2020) Possible protective role of $17 \beta$-estradiol against COVID-19. J Allergy Infect Dis 1:38-48

34. Waldron JL, Ashby HL, Cornes MP et al (2013) Vitamin D: a negative acute phase reactant. J Clin Pathol 66:620-622

35. Ilie PC, Stefanescu S, Smith L (2020) The role of vitamin D in the prevention of coronavirus disease 2019 infection and mortality. Aging Clin Exp Res 32:1195-1198

36. Isaia G, Medico E (2020) Associations between hypovitaminosis D and COVID-19: a narrative review. Aging Clin Exp Res 32:1879-1881

Publisher's Note Springer Nature remains neutral with regard to jurisdictional claims in published maps and institutional affiliations. 